\title{
Effect of Flexible Half-dose Gonadotropin-releasing Hormone Antagonist Protocol on in Vitro Fertilization Outcome in Predicted Normal Responder: A Study Protocol for a Multicentered, Randomized, Non-inferiority, Parallel Controlled Trial
}

\section{Ying-Jie Ma}

Shandong University of Traditional Chinese Medicine

Jing-Yan Song

Shandong University of Traditional Chinese Medicine

Meng-Yao Li

Shandong University of Traditional Chinese Medicine

Zhengao Sun ( $\nabla$ sunzhengao77@126.com )

The affiliated hospital Shandong University of Traditional Chinese Medicine https://orcid.org/00000002-9723-1213

\section{Research Article}

Keywords: Gonadotropin-releasing hormone antagonist, In vitro fertilization-embryo transfer, Half-dose GnRH-ant protocol, Predicted normal responder, Randomized controlled trial

Posted Date: October 29th, 2021

DOl: https://doi.org/10.21203/rs.3.rs-871729/v1

License: (c) (i) This work is licensed under a Creative Commons Attribution 4.0 International License. Read Full License 


\section{Abstract}

\section{Background}

Gonadotropin-releasing hormone antagonists (GnRH-ant) are widely used in current in vitro fertilizationembryo transfer (IVF-ET), however, whether the lowest daily dose of $\mathrm{GnRH}$-ant is individualized remains unknown. Due to the negative effect of $\mathrm{GnRH}$-ant on endometrial receptivity, lessening the amount of $\mathrm{GnRH}$-antagonists used during controlled ovarian stimulation may be helpful for embryo implantation. As such, a randomized controlled study is essential to validate the feasibility and efficacy of daily GnRH-ant dose reduction to $0.125 \mathrm{mg}$ geared towards providing scientific evidence for guidance in clinical practice.

Methods

In total, 620 infertile women undergoing in vitro fertilization will be enrolled in the multicentered, randomized, parallel controlled trial. Based on a computer-generated random list, they will be randomly and equally subdivided into half-dose $\mathrm{GnRH}$-ant group or conventional-dose $\mathrm{GnRH}$-ant group. The primary outcome is ongoing pregnancy i.e., intrauterine pregnancy diagnosed by pelvic ultrasonography at more than 12 weeks of gestation accompanied by normal fetal heartbeats. Secondary outcomes include cycle cancellation, premature luteinizing hormone surge, positive pregnancy, embryo implantation rate, clinical pregnancy, early spontaneous abortion, and live birth. The intention-to-treat and per protocol analyses will be used to initially analyze the difference in ongoing pregnancy rate between the two groups, while the multiple imputation method was used to handle missing values in the data.

Discussion

At present, no randomized controlled trials (RCTs) have been performed on the use of the half-dose GnRH-ant protocol $(0.125 \mathrm{mg} / \mathrm{d})$ to improve reproductive outcomes of IVF-ET in predicted normal responder, compared to conventional-dose $\mathrm{GnRH}$-ant protocol $(0.25 \mathrm{mg} / \mathrm{d})$. Half-dose $\mathrm{GnRH}$-ant protocol might provide a suitable clinical solution for predicted normal responder undergoing IVF treatment. Thus, it is critical to conduct a well-designed RCT to evaluate the impact of a half-dose $\mathrm{GnRH}$-ant protocol on the reproductive outcomes of IVF-ET in predicted normal responder.

Trial registration

This study was registered in the Chinese Clinical Trials Registry Platform on August 29, 2020. (chictr.org.cn; identifier: ChiCTR2000037629).

\section{Strengths And Limitations}

(1) The first parallel controlled trial on the minimum daily dose of GnRH-ant for individualized flexible use; 
(2) The first trial aims to confirm the feasibility and efficacy of daily GnRH-ant dose reduction to 0.125 $\mathrm{mg}$ to provide a scientific basis for improving the success rate of in vitro fertilization-embryo transfer;

(3) The study is a prospective, multicenter, randomized controlled trial with more sufficient clinical evidence;

(4) Researchers, doctors, and participants cannot be blinded to treatment allocation.

\section{Background}

Gonadotropin-releasing hormone antagonists (GnRH-ant) protocols are widely used in present IVF-ET treatment. At the same time, the ESHRE guidelines strongly propose the use of $\mathrm{GnRH}$-ant protocol during controlled ovarian hyperstimulation $(\mathrm{COH})$ in predicted normal responders ${ }^{[1]}$. Unlike gonadotropinreleasing hormone agonist ( $\mathrm{GnRH}-\mathrm{a}$ ) protocol, $\mathrm{GnRH}$-ant protocol has several advantages, including shortening the treatment time, reducing gonadotropin dosage, and the occurrence of severe ovarian hyperstimulation syndrome (OHSS), as well as preventing pituitary over-suppression and flare-up effects

[2]. Although the $\mathrm{GnRH}$-ant protocol has been reported to be comparable to the $\mathrm{GnRH}$-a protocol in terms of cumulative live birth rates ${ }^{[3,4]}$, other studies have shown that the $\mathrm{GnRH}$-ant protocol results in decreased clinical pregnancy rates ${ }^{[5,6]}$. A recent systematic review revealed that frozen-thawed embryo transfer (FET) had no benefit on live birth rate compared to fresh embryo transfer, thus, the "freeze-all" strategy was not recommended for predicted normal responder due to the increased risk of preeclampsia [7]. Additionally, a number of studies have shown that $\mathrm{GnRH}$ antagonists are associated with decreased frequencies of ongoing pregnancy in fresh embryo transfer cycles when compared to $\mathrm{GnRH}$-a long protocol, whereas the detrimental effects of $\mathrm{GnRH}$-ant on endometrial receptivity were the primary reasons for the poor pregnancy rate ${ }^{[5,8,9]}$.

To minimize the detrimental effect of $\mathrm{GnRH}$-ant on endometrial receptivity, reproductive doctors have attempted to improve the $\mathrm{GnRH}$-ant protocol by establishing a minimum dose of $\mathrm{GnRH}$-ant. So far, starting on day 6 of ovarian stimulation (OS), $0.25 \mathrm{mg}$ of cetrorelix per day is thought to maintain luteinizing hormone $(\mathrm{LH})$ levels within a safe range, a dose frequently used in clinical practice ${ }^{[10-13]}$. Nevertheless, a few studies have shown that limiting the daily dosage of $\mathrm{GnRH}$-ant to $0.125-0.2 \mathrm{mg}$ is similarly beneficial. ${ }^{[14-16]}$. Additionally, Wang and his colleges reported that routine $\mathrm{GnRH}$-ant administration was not necessary during ovarian stimulation, since 87.2 percent of patients did not need any $\mathrm{GnRH}$-ant mostly during $\mathrm{COH}$ cycle ${ }^{[17]}$. As a result, the minimal effective dosage of $\mathrm{GnRH}$-ant remains controversial.

Hence, we assume that there exists no specific $\mathrm{GnRH}$-ant dose suitable for each participant, therefore, it is absolutely critical that the dosage of GnRH should be tailored to the needs of each patient. Based on the definition of premature LH surge, i.e., serum LH level $\geq 10 \mathrm{IU} / \mathrm{L}$ and progesterone $(P)$ level $\geq 1.0 \mathrm{ng} / \mathrm{ml}$, and the less effective $\mathrm{GnRH}$-ant dose described in previous studies ${ }^{[18-20]}$, we tried a novel and flexible half-dose $\mathrm{GnRH}$-ant protocol in a small number of patients and design this randomized controlled trial in 
which the daily $\mathrm{GnRH}$-ant dose will be reduced to $0.125 \mathrm{mg}$. The daily $\mathrm{GnRH}$-ant dose will be increased to $0.25 \mathrm{mg}$ until the trigger day if the LH level rises above $10 \mathrm{IU} / \mathrm{L}$.

\section{Methods/design Study design}

A multicenter, prospective, randomized parallel controlled trial will be conducted in four reproductive centers in China Mainland. The flow chart for this study is shown in Fig. 1, and the study process schedule is shown in Table 1. 
TABLE 1 | Schedule of the study process (SPIRIT Figure).

\section{STUDY PERIOD}

\section{Enrolment Allocation Post-allocation}

Closeout

TIMEPOINT**

0 days

$\begin{array}{lllll}1-6 & 6-15 & 16- & 6-12 & 13-40 \\ \text { days } & \text { days } & \begin{array}{l}30 \\ \text { days }\end{array} & \text { weeks } & \text { weeks }\end{array}$

\section{ENROLMENT:}

Inquire medical

$x$

history

\section{Basic information $\quad x$ \\ registration (E2, LH, \\ FSH, P, BMI, etc.)}

Vaginal ultrasound $\quad x$

and biochemistry

Randomization

$\mathrm{X}$

INTERVENTIONS:

$0.125 \mathrm{mg}$ of

cetrorelix

$0.25 \mathrm{mg}$ of cetrorelix

ASSESSMENTS:

Cycle cancellation,

Premature LH surge

Positive pregnancy,

Embryo implantation

rate

Clinical pregnancy

Early Spontaneous

Abortion

Ongoing pregnancy

Live birth

Objectives

$\mathrm{GnRH}$-ant is believed to be negatively correlated with the capacity for endometrium receptivity. When using the $\mathrm{GnRH}$-ant protocol, lowering $\mathrm{GnRH}$-ant doses during controlled OS can aid in embryo implantation. Nonetheless, whether the minimum daily dose of $\mathrm{GnRH}$-ant should be individualized 
remains unknown. Thus, this study aims to resolve the concerns about the efficacy and safety of administering $\mathrm{GnRH}$-ant a regular dose of $0.125 \mathrm{mg}$.

\section{Participants}

\section{Inclusion criteria}

Initial IVF/ICSI treatment; Age $<40$ years; predicted normal responder, i.e., the number of basal antral follicles count (AFC) was between 5 and $15^{\text {[21]. }}$.

\section{Exclusion criteria}

Couples with chromosomal abnormalities; history of oophorectomy; history of pelvic chemoradiotherapy; polycystic ovary (PCO) or polycystic ovary syndrome (PCOS); ovarian endometriotic cysts requiring surgery; untreated hydrosalpinx; history of recurrent implantation failure (i.e. the failure to achieve a viable pregnancy, following a greater than 3 embryo transfers with high-quality embryos) and recurrent pregnancy loss (i.e. couples with two or more consecutive spontaneous miscarriages); embryo transfer cancelled for patient personal reasons; patients requiring egg donation; embryo transfer cancelled for uterine or endometrial abnormalities, such as endometriosis, uterine fibroids, endometrial polyps, or intrauterine adhesions.

\section{Recruitment}

Detailed history, transvaginal ultrasound, and biochemistry will be required before $\mathrm{COH}$, and if inclusion and exclusion criteria are met, the patient will be contacted by a research assistant, where the study details will be explained to her, then invited to join the study. Each individual may willingly attend the study and will be provided a detailed explanation of the trial. At the same time, their permission will be sought before being recruited into the study. Patients must sign a written informed consent.

\section{Interventions}

Cetrorelix acetate can be provided in two groups: some patients will be treated with a regular dose, whereas others will get a low dosage. In the $\mathrm{GnRH}$-ant half-dose group, on day five or six of $\mathrm{COH}$, $0.125 \mathrm{mg}$ of $\mathrm{GnRH}$-ant (Cetrorelix acetate, Merck Serono, Switzerland) will be subcutaneously injected daily. Patients will be subjected to serological testing at 1- or 2-days interval, and once LH levels exceed $10 \mathrm{mIU} / \mathrm{mL}$, the daily cetrorelix dose will be adjusted to $0.25 \mathrm{mg}$ until the trigger day. In the $\mathrm{GnRH}$-ant conventional-dose group, on day five or six of $\mathrm{COH}, 0.25 \mathrm{mg}$ of $\mathrm{GnRH}$-ant will be subcutaneously injected daily until the trigger day.

\section{In vitro fertilization procedure}

OS begins on the second day of menstruation, and all patients will be provided recombinant folliclestimulating hormone (r-FSH) (75U, Gonal-F, Merck Serono, Switzerland). The initial FSH dose was established by the physician based on age, AFC, basal FSH, and body mass index (BMI), usually ranging 
from 150-225 IU/day. This dose is adjusted every 2-3 days, depending on ovarian response, i.e., serum estradiol levels and follicular growth under ultrasound monitoring. Then, all patients will be treated with GnRH-ant $(0.25 \mathrm{mg}$, Cetrorelix acetate, Merck Serono, Switzerland) from day 5 or 6 of $\mathrm{COH}$ until the day before the trigger. Finally, 250 $\mu$ g of recombinant human chorionic gonadotropin (r-hCG, Merck Serono, Switzerland) will be injected when the mean diameter of $\geq 3$ follicles reach $17 \mathrm{~mm}$. After 35 to 37 hours of $\mathrm{r}$-hCG injection, oocyte retrieval will be guided by transvaginal ultrasound. Notably, intracytoplasmic sperm injection (ICSI) will be performed only in cases of severe oligozoospermia and asthenozoospermia

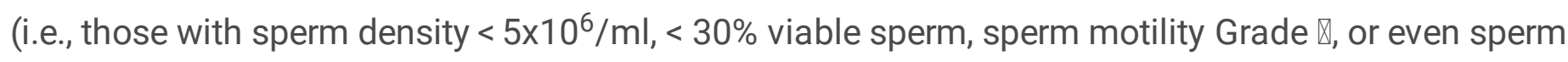
immotile but confirmed with viable sperm by eosin staining).

In our present study, only fresh embryo(s) will be transferred. On day three after oocyte recovery, embryo transfer will commence. All patients should undergo embryo transfer on the third day after oocyte retrieval, except for the following conditions: (1) serum $E_{2}>5000 \mathrm{pg} / \mathrm{mL}$ on the trigger day; (2) number of

oocytes retrieved $\geq 15$; (3) OHSS; (4) serum $\mathrm{P}>1.75 \mathrm{ng} / \mathrm{mL}$ before the trigger day [22]; or (5) patients who violate informed consents and waived fresh embryo transfer. No more than 2 embryos will be transferred at a time.

Luteal support will start on the first day after oocyte retrieval with $8 \%$ micronized vaginal progesterone gel 90 mg (Crinone $\AA$; Central Pharma Ltd, UK) once daily and dydrogesterone (Duphaston $\AA ;$; Abbott Biologicals, Netherlands) $20 \mathrm{mg}$ orally daily until 10 weeks of gestation.

\section{Primary outcome}

Ongoing pregnancy: intrauterine pregnancy confirmed by pelvic ultrasonography at more than 12 weeks of gestation accompanied by normal fetal heartbeats.

\section{Secondary outcomes}

(1) Cycle cancellation: premature ovulation, absence of follicular development, failure to pick up eggs, and personal reasons;

(2) Premature LH surge: serum LH level $\geq 10 \mathrm{IU} / \mathrm{L}$ or $\mathrm{P}$ level $\geq 1.0 \mathrm{ng} / \mathrm{ml}$;

(3) Positive pregnancy or chemical pregnancy: 14 days after embryo transfer, serum $\beta$-hCG $\geq 10 \mathrm{mlU} / \mathrm{mL}$;

(4) Embryo implantation: the number of gestational sacs identified via pelvic ultrasonography divided by the total number of embryos transferred;

(5) Clinical pregnancy: intrauterine gestational sac with fetal heartbeat was identified by B-mode ultrasound at 6 gestational weeks;

(6) Early spontaneous abortion: spontaneous termination of pregnancy occurring in less than 12 weeks of gestation; 
(7) Live birth: delivery of a neonate with a gestational age $\geq 20$ weeks and heartbeat and respiration.

(8) Pregnancy related complications: gestational diabetes, gestational hypertension, preeclampsia, and preterm delivery.

\section{Randomization}

When patients volunteering to participate in the study will be joined, stratified block randomization will be used, i.e., stratified by study site. The block length will be set to four. The random number will be generated then block randomization will be performed based on $\mathrm{R}$ language version 3.5.1. The proportion of subjects in the two groups is 1:1. The specific grouping details are mastered by the drug management center and the statisticians, and the subjects will be randomly assigned to one of the following two groups: (1) $\mathrm{GnRH}$-ant half-dose group: On the 6 th day of $\mathrm{COH}, 0.125 \mathrm{mg}$ cetrorelix was subcutaneously injected daily. Patients underwent serological testing at 1- or 2-days interval, and once LH levels exceed $10 \mathrm{mIU} / \mathrm{mL}$, the daily cetrorelix dose will be adjusted to $0.25 \mathrm{mg}$ until the trigger day; (2) $\mathrm{GnRH}$-ant conventional-dose group: On the 6 th day of $\mathrm{COH}, 0.25 \mathrm{mg}$ cetrorelix is injected subcutaneously daily until the trigger day.

\section{Blinding}

Because of the nature of the trial, neither the researchers nor the patients will be blinded. Nevertheless, the embryologists performing the quality assessment must be blinded to the grouped interventions.

\section{Estimation of Sample Size}

PASS software version 11.0 (NCSS, LLC. Kaysville, Utah, USA.) will be used to calculate the sample size of the two groups. It is worth noting that based on the data of our reproductive center at the time of the trial design, the ongoing pregnancy rate of each fresh embryo transfer was about $30 \%$. Moreover, it is calculated that the sample size of the $\mathrm{GnRH}$-ant low dose group (intervention group) and $\mathrm{GnRH}$-ant conventional-dose group (control group) is 279 cases, with a total sample size of 558 cases. The equivalent detection ability of $80 \%$ was achieved to provide $80 \%$ certainty and to demonstrate noninferiority with a margin of $10 \%$ of the main outcome of ongoing pregnancy between the two groups at bilateral $a=0.05$. The Calculation hypothesis was the bilateral $Z$ test. This trial sets the dropout rate at $10 \%$. The overall sample size of the two groups is 620 , with 310 in each group.

\section{Statistical Analysis}

Intention-to-treat and per protocol analyses will be used to analyze the difference of persistent pregnancy rate between the two groups in the main analysis. The multiple interpolation method will be used to deal with the missing values in the data of this study.

Demographic characteristics and baseline data are compared between the two groups. Students' t-test is used to compare continuous variables, while the $\chi 2$ test is used to compare classified variables.

Additionally, a subgroup analysis-age $\leq 35$ years old vs $>35$ years old will be conducted to further explore the effect of flexible half-dose $\mathrm{GnRH}$-ant regimen on in vitro fertilization outcomes in predicted normal 
responders. All the data will be statistically analyzed using SPSS version $26.0, \mathrm{P}<0.05$ is considered as statistically significant.

\section{Feasibility analysis}

Regarding the feasibility of research ideas, this trial considers the population with predicted normal response and a wide range of beneficiary populations. Moreover, a recent meta-analysis including eleven high-quality randomized controlled trials (RCTs) suggested that the whole embryo freezing strategy is not recommended for predicted normal responder due to the increased risk of preeclampsia compared to fresh embryo transfer. The adverse effect of traditional $\mathrm{GnRH}$-ant protocol on the clinical pregnancy rate of fresh embryo transfer is the original intention of this trial. This trial sights to improve the clinical pregnancy rate in this population by reducing the dosage of $\mathrm{GnRH}$-ant to alleviate its adverse effect on the endometrium.

The project applicant, Zhen-Gao Sun, MD, presided over several National Natural Science Foundation of China. Also, the applicant presided over and participated in the implementation of several multicenter RCTs nationwide, with good scientific research literacy; the applicants and researchers had graduate degree or above and a good cooperation relationship. Besides, the members of the research group received systematic training through the National Natural Science Foundation of China and provincial projects, mastered the knowledge of protocol design, reproductive medicine, medical statistics, and other aspects of the study of this project, they also had strong technical strength.

\section{Data Entry and Quality Control}

Embryo lab data will be collected on day 3 or 5 post oocyte retrieval. A As per the study design, participants' follow-up data will comprise information from the time they enrolled in the study and for the next year. Furthermore, case report forms from patients will be obtained for data input. Investigators will also be needed to determine the data accuracy levels (first and second levels), since both levels will involve data validation and monitoring, which will be performed on a regular basis by the Data Monitoring Committee (DMC) during the study. On a daily basis, data will be backed up to another computer in the same physical area as the server.

\section{Patient and public involvement}

Patients and the public are not involved in developing the research questions nor the study design. Moreover, they do not participate in the recruitment exercise of the study. Our results will be disseminated to participants and their families via telephone and patient organization platforms.

\section{Existing Conditions and Work Basis}

Based on the IVF-ET technology platform of the Reproductive Center, Affiliated Hospital of Shandong University of Traditional Chinese Medicine (TCM), the project research team performed IVF-ET for more than 10 years for the earliest TCM unit to start IVF technology. This was approved by the Ministry of Health in China, and implemented IVF/ICSI about 2,000 cycles per year. 
The members of the research group had a reasonable echelon, high quality, and a good relationship with many years of cooperation, in completion of many RCTs, and well completed the research work of this topic.

\section{Ethics and Dissemination}

Clinicians received written informed consent from each patient participating in the study before the study commences. This study was approved by the Ethics Committee of Reproductive Medicine Ethics Committees of the Affiliated Hospital of Shandong University of TCM (SDTCM-RM-2020.611) in Jinan City, Shandong Province, China. The findings of this study were widely disseminated via conference papers, research reports, and academic publications.

Likewise, data will be electronically inputted, saved, and encrypted in secure computer files available only to the study's research team and investigators. Additionally, the original study forms will be kept and maintained at the study location for three years after the end of the study. Also, the investigators will permit Institutional Review Board/Independent Ethics Committee (IRB/IEC) evaluations, audits, and monitoring of the trial's data sources and required documents. Subsequently, participants will be asked to contact their physician with any study-related concerns.

\section{Trial status}

This trial is at version 1.7, 29 August 2020 (ChiTR). The actual study start date was 1 April 2021 and the anticipated study end date is 31 December 2021. The recruitment start date was 15 May2021; the anticipated recruitment end date is 31 October 2021.

\section{Discussion}

Premature LH surge is characterized as an LH level of $10 \mathrm{IU} / \mathrm{L}$ and a $\mathrm{P}$ level of $1.0 \mathrm{ng} / \mathrm{ml}$, and is produced by numerous follicle recruitment and fast estrogen rise. ${ }^{[23,24]}$. Premature LH surge during ovarian stimulation in IVF is detrimental to egg development, resulting in luteinization and premature ovulation, leading to poor reproductive results and even cycle cancellation ${ }^{[4]}$. Although this is still disputed, pregnancy rates with the $\mathrm{GnRH}$-ant protocol have been reported to be lower than those with the $\mathrm{GnRH}$ agonist protocol, with reduced endometrial receptivity being the primary reason for this discrepancy. Reduced GnRH-ant dosages have been used by physicians in reproductive clinics to try to obtain acceptable pregnancy chances. However, the optimal daily dosage of $\mathrm{GnRH}$-ant continues to be hotly debated. At the moment, $0.25 \mathrm{mg}$ of Cetrorelix per day starting on day 5 or 6 of ovarian stimulation is considered the standard $\mathrm{GnRH}$-ant protocol, and the $\mathrm{LH}$ level may be controlled within a safe range with this procedure. According to some other studies, limiting the $\mathrm{GnRH}$-ant dosage to $0.125-0.2 \mathrm{mg}$ per day is also beneficial. Daily GnRH-ant dosage requirements for the $\mathrm{GnRH}$-ant regimen, we believe, should be determined on an individualized level. Based on previous studies ${ }^{[15,25]}$, we tried to reduce the initial daily dose of cetrorelix to $0.125 \mathrm{mg}$, which was then individually adjusted to $0.25 \mathrm{mg}$ based on LH levels during controlled ovarian stimulation and as a $\mathrm{GnRH}$-ant half-dose group, furthermore individuals 
assigned to the $\mathrm{GnRH}$-ant conventional-dose group will receive $0.25 \mathrm{mg}$ cetrorelix daily for the duration of the controlled ovarian stimulation.

A panel of seasoned professionals will perform this randomized controlled study. Based on our experience in conducting RCTs, this study will demonstrate that GnRH-ant half-dose group has a convincing effect on improving reproductive outcomes in patients with predicted normal response, compared to $\mathrm{GnRH}$-ant conventional-dose group.

\section{Abbreviations}

GnRH-ant: gonadotropin-releasing hormone antagonists; IVF-ET: in vitro fertilization-embryo transfer; FET: frozen-thawed embryo transfer; RCT: randomized controlled trial; $\mathrm{COH}$ : controlled ovarian hyperstimulation; OHSS: ovarian hyperstimulation syndrome; OS: ovarian stimulation; LH: luteinizing hormone; AFC: antral follicle count; PCOS: polycystic ovary syndrome; FSH: follicle-stimulating hormone; BMI: body mass index; r-hCG: recombinant human chorionic gonadotropin; ICSI: intracytoplasmic sperm injection; CRF: case report form; TCM: traditional Chinese medicine.

\section{Declarations}

\section{Ethics approval and consent to participate}

This study was approved by the Reproductive Medicine Ethics Committee of the Affiliated Hospital of Shandong University of TCM (SDTCM-RM-2020.611) in Jinan, Shandong, China. All participate will be required to sign written informed consent.

\section{Consent for publication}

Not applicable.

\section{Availability of data and material}

Not applicable.

\section{Competing interests}

None of the authors have a conflict of interest to declare with regard to this study.

\section{Funding}

This work was supported by the National Natural Science Foundation of China (No. 81874484). The funder was not involved in the study design, collection, analysis, interpretation of data, the writing of this article or the decision to submit it for publication.

\section{Authors' contributions}


Zhen-Gao Sun and Jing-Yan Song designed and supervised this study. Ying-Jie Ma wrote the manuscript. Meng-Yao Li and Jing-Yan Song modified the manuscript. Ying-Jie Ma made significant contributions to the sample size estimation and performed the statistical analysis.

\section{Acknowledgments}

We appreciate Jing-Yan Song and Zhen-Gao Sun for their valuable support in designing this study.

\section{References}

1. Ovarian Stimulation T, Bosch E, Broer S, Griesinger G, Grynberg M, Humaidan P, Kolibianakis E, Kunicki M, La Marca A, Lainas G, Le Clef N, Massin N, Mastenbroek S, Polyzos N, Sunkara SK, Timeva T, Toyli M, Urbancsek J, Vermeulen N, Broekmans F. ESHRE Guideline: ovarian stimulation for IVF/ICSI (dagger). Hum Reprod Open 2020, 2020 (2): hoaa009.

2. Copperman AB, Benadiva C. Optimal usage of the $\mathrm{GnRH}$ antagonists: a review of the literature. Reprod Biol Endocrinol 2013, 11:20.

3. Xiao JS, Su CM, Zeng XT. Comparisons of GnRH antagonist versus GnRH agonist protocol in supposed normal ovarian responders undergoing IVF: a systematic review and meta-analysis. PLoS One 2014, 9 (9): e106854.

4. Al-Inany HG, Youssef MA, Ayeleke RO, Brown J, Lam WS, Broekmans FJ. Gonadotrophin-releasing hormone antagonists for assisted reproductive technology. Cochrane Database Syst Rev 2016, 4: CD001750.

5. Lambalk CB, Banga FR, Huirne JA, Toftager M, Pinborg A, Homburg R, van der Veen F, van Wely M. GnRH accounting versus long agonist protocols in IVF: a systematic review and meta-analysis for patient antagonist type. Hum Reprod Update 2017, 23 (5): 560-579.

6. Al-Inany $\mathrm{H}$, Aboulghar M. GnRH antagonist in assisted reproduction: a Cochrane review. Hum Reprod 2002, 17 (4): 874-885.

7. Roque M, Haahr T, Geber S, Esteves SC, Humaidan P. Fresh versus elective frozen embryo transfer in IVF/ICSI cycles: a systematic review and meta-analysis of reproductive outcomes. Hum Reprod Update 2019, 25 (1): 2-14.

8. Xu B, Zhou M, Wang J, Zhang D, Guo F, Si C, Leung PCK, Zhang A. Increased AlF-1-mediated TNF-alpha expression during implantation phase in IVF cycles with $\mathrm{GnRH}$ antagonist protocol. Hum Reprod 2018, 33 (7): $1270-1280$.

9. Xu B, Wang J, Xia L, Zhang D, Wu X, Zhang A. Increased Uterine NK cell numbers and numbers in expression during the implantation phase in IVF Cycles with GnRH Antagonist Protocol. Sci Rep 2017, 7:39912. 
10. Marci R, Graziano A, Lo Monte G, Piva I, Soave I, Marra E, Lisi F, Moscarini M, Caserta D. GnRH antagonists in assisted reproductive techniques: a review on the Italian experience. Eur Rev Med Pharmacol Sci 2013, 17 (7): 853-873.

11. Mokhtar S, Sadeghi MR, Akhondi MM, Zafardoust S, Badenush B, Fatemi F, Nazari F, Kamali K, Mohammadzade A. ART Outcomes in GnRH Antagonist Protocol (Flexible) and Long GnRH Agonist Protocol in Randomized Patients with Polycystic Ovary Syndrome: A Early Clinical Trial. J Reprod Infertil 2015, 16 (3): 148-154.

12. Kolibianakis EM, Venetis CA, Kalogeropoulou L, Papanikolaou E, Tarlatzis BC. Fixed versus flexible gonadotropin-releasing hormone antagonist administration in vitro fertilization: a randomized controlled trial. Fertil Steril 2011, 95 (2): 558-562.

13. Hsieh YY, Chang CC, Tsai HD. Comparisons of different dosages of gonadotropin-releasing hormone $(\mathrm{GnRH})$ antagonist, short-acting form and single, half-dose, long-acting form of GnRH agonist during controlled ovarian hyperstimulation and in vitro fertilization. Taiwan J Obstet Gynecol 2008, 47 (1): 6674.

14. Chang YL, Hsieh YY, Tsai HD. Preliminary report on the effect of a lower dose of gonadotropinreleasing hormone antagonist (cetrorelix) on ovarian hyperstimulation in lower-weight Asian women. Taiwan J Obstet Gynecol 2006, 45 (4): 317-320.

15. Kerimoglu OS, Ercan CM, Keskin U, Korkmaz C, Duru NK, Ergun A. Effect of a low dose of gonadotropin-releasing hormone antagonist on in vitro fertilization outcomes. Arch Gynecol Obstet 2013, 288 (3): 691-695.

16. Chen HJ, Lin YH, Hsieh BC, Seow KM, Hwang JL, Tzeng CR. Is a lower dose of cetrorelix acetate effective for prevention of LH surge during controlled ovarian hyperstimulation? J Assist Reprod Genet 2006, 23 (6): 289-292.

17. Wang HL, Lai HH, Chuang TH, Shih YW, Huang SC, Lee MJ, Chen SU. A Patient Friendly Corifollitropin Alfa Protocol without Routine Pituitary Suppression in Normal Responders. PLoS One 2016, 11 (4): e0154123.

18. Albano C, Smitz J, Camus M, Riethmuller-Winzen H, Van Steirteghem A, Devroey P. Comparison of different doses of gonadotropin-releasing hormone Cetrorelix during controlled ovarian hyperstimulation. Fertil Steril 1997, 67 (5): 917-922.

19. Albano C, Felberbaum RE, Smitz J, Riethmuller-Winzen H, Engel J, Diedrich K, Devroey P. Ovarian stimulation with HMG: results of a prospective phase III European study comparing the einizing hormonereleasing hormone (LHRH) -cetrorelix and the LHRH-agonist antagonist buserelin. European Cetrorelix Study Group. Hum Reprod 2000, 15 (3): 526-531. 
20. Engel JB, Ludwig M, Felberbaum R, Albano C, Devroey P, Diedrich K. Use of cetrorelix in combination with clomiphene citrate and gonadotrophins: a friendly approach to 'IVF'? Hum Reprod 2002, 17 (8): 2022-2026.

21. Yeung T, Chai J, Li R, Lee V, Ho PC, Ng E. A double-blind randomized controlled trial on the effect of dehydroepiandrosterone on ovarian reserve markers, ovarian response, and number of responders in normal ovarian oocytes. BJOG 2016, 123 (7): 1097-1105.

22. Xu B, Li Z, Zhang H, Jin L, Li Y, Ai J, Zhu G. Serum progesterone level effects on the outcome of in vitro fertilization in patients with different ovarian response: an analysis of more than 10,000 cycles. Fertil Steril 2012, 97 (6): 1321-1327.

23. Olivennes F, Belaisch-Allart J, Emperaire JC, Dechaud H, Alvarez S, Moreau L, et al. Prospective, randomized, controlled study of in vitro fertilization-embryo transfer with a single dose of a luteinizing hormone-releasing hormone (LH-RH) antagonist (cetrorelix) or a depot formula of an LH-RH agonist (triptorelin). Fertil Steril. 2000, 73 (2): 314-320.

24. Engel JB, Felberbaum RE, Eilers W, Polack S, Ortmann O, Diedrich K. Clomiphene-induced LH surges and cetrorelix. Reprod Biomed Online. 2002, 5 (2): 109-111.

25. Zhang D, Xia L, Xu H, et al. Flexible Low-Dose GnRH Antagonist Protocol Is Effective in Patients With Sufficient Ovarian Reserve in IVF. Front Endocrinol (Lausanne). 2018; 9:767.

\section{Figures}




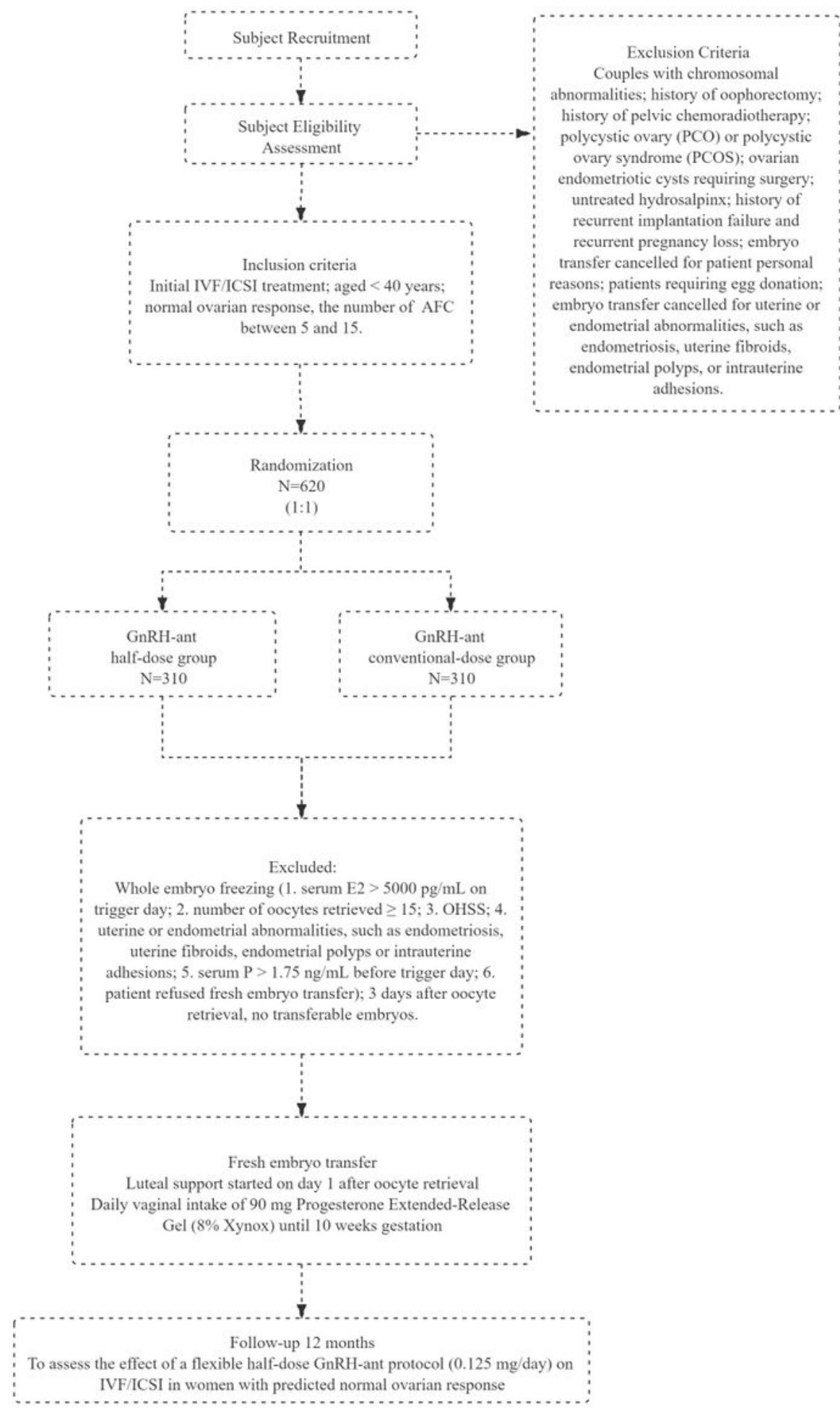

\section{Figure 1}

Flowchart showing the study process.

\section{Supplementary Files}

This is a list of supplementary files associated with this preprint. Click to download. 
- SPIRITChecklist.docx

Page 16/16 